\title{
Comparison of NS-1 Antigen Detection by ICT and ELISA for Evaluating Acute Dengue
}

\author{
S. Mahesh Kumar and S. Sheethal* \\ Department of Microbiology, Karnataka Institute of Medical Sciences, KIMS campus, \\ Vidyanagar, Hubballi- 580022, Karnataka, India \\ *Corresponding author
}

\begin{tabular}{|l|}
\hline Ke y w or d s \\
Dengue fever, NS1 \\
ICT, NS1 ELISA, \\
IgM MAC-ELISA \\
\hline Article Info \\
\hline $\begin{array}{l}\text { Accepted: } \\
\text { 28 January } 2018 \\
\text { Available Online: } \\
\text { 10 February } 2018\end{array}$ \\
\hline
\end{tabular}

A B S T R A C T

Dengue is an important mosquito-borne viral disease of humans. Rapid and easy diagnosis of dengue can assist patient triage and care-management. Serological detection of denguespecific IgM has been the main stay of diagnosis. The detection of dengue NS1 offers a faster presumptive diagnosis. (1) To compare the rapid immunochromatographic card and ELISA methods of NS1 antigen detection. (2) To detect dengue-specific IgM antibodies by MAC-ELISA. Blood specimens collected from patients having febrile illness clinically diagnosed as having dengue fever as per WHO criteria were subjected to detection of NS1 antigen by rapid ICT cards and ELISA, and IgM antibody by MAC-ELISA. Among 116 suspected dengue cases, $25 \%$ were positive by NS1 ICT, $29.3 \%$ were positive by NS1 ELISA and $37.9 \%$ were positive by IgM MAC-ELISA. The sensitivity of NS1 ICT was $52.27 \%$ and specificity was $91.66 \%$ whereas sensitivity for ELISA was $56.81 \%$ and specificity was $87.5 \%$. There was no statistical significance between duration of illness and application of the tests. Overall prevalence of dengue infection was $45.7 \%$. NS1 ELISA showed a slightly better sensitivity but specificity of NS1 ICT was higher. However, for early diagnosis and management of acute dengue, both NS1 antigen and IgM antibody detection tests need to be used in conjunction.

\section{Introduction}

Benjamin Rush described "break bone fever" when dengue made its debut in 1780. This mosquito borne fast emerging viral infection manifests in four serotypes capable of causing dengue fever (DF), dengue hemorrhagic fever (DHF), and dengue shock syndrome (DSS), poses an increasingly perilous situation due to lack of antiviral drugs (Kumar et al., 2010).

Dengue is almost endemic throughout India (Kulkarni et al., 2011). India is one of the seven identified countries in the South-East
Asia region regularly reporting incidence of $\mathrm{DF} / \mathrm{DHF}$ outbreaks. The first confirmed report of dengue infection in India was during the 1940s. Since then, newer states have joined in reporting the disease. Dengue strikes in epidemic proportions often inflicting severe morbidity and mortality, in urban and rural environments alike (Kumar et al., 2010).

Establishing a diagnosis of acute dengue virus infection during the first few days after manifestation of clinical symptoms becomes important for providing timely information to manage the patients, and to instate early public 
health control measures to prevent dengue outbreaks. Presently, the basic methods used by most laboratories for the diagnosis of dengue virus infection include viral isolation, detection of viral genomic sequence by nucleic acid amplification technology assay (RT-PCR), and the detection of dengue virusspecific IgM antibodies by the IgM-capture enzyme linked immunosorbent assay (MACELISA) and/or the rapid dengue immunochromatographic test (ICT) (Padhi et al., 2014). Antigen detection, particularly nonstructural protein 1 (NS1), is another method that can be employed for diagnosing acute dengue (Watthanaworawit et al., 2011).

In this following study, we have evaluated rapid dengue NS1 immunochomatographic card test (ICT), NS1 enzyme linked immunosorbent assay (ELISA) and dengue specific IgM antibody detection by Immunoglobulin $\mathrm{M}$ capture ELISA (MACELISA) for early and correct diagnosis of dengue virus infection in a tertiary care hospital.

\section{Materials and Methods}

The study was conducted at a tertiary care hospital from January 2014 to December 2014 after permission was granted by the institutional ethical committee. Two sets of serum samples were collected from a total of 116 patients suffering from acute febrile illness clinically suspected of dengue fever. The first sample was collected between 4-7 days of fever and the following sample was collected after 4 days of fever onset up to day 10. The test kits used were Denguecheck Combo supplied by Zephyr Biomedicals, Goa, India; Dengue NS1 Ag Microlisa supplied by J Mitra \& Co. Pvt. Ltd, New Delhi, India; and Dengue-IgM antibody capture ELISA supplied by Arbovirus Diagnostics, NIV, Pune, India. The tests were performed strictly as per the manufacturer's instructions. This evaluation was done keeping in mind the availability of these tests in a peripheral centre for diagnosing dengue fever. NS1 positivity has been proved beyond doubt to be negligible in this group which warranted non-inclusion of healthy individuals as controls in our study.

\section{Results and Discussion}

We observed an overall prevalence of dengue infection at $45.7 \%$. NS1 antigen was detected in $29(25 \%)$ patients by ICT and in $34(29.3 \%)$ patients by ELISA. IgM ELISA detected 44 (37.93\%) positive cases (Table 1$)$. This study found the sensitivity of rapid dengue NS1 detection kits to be $52.3 \%$ with a specificity of $91.7 \%$ and the sensitivity and specificity of dengue NS1 ELISA was at $56.8 \%$ and $87.5 \%$ respectively in comparison to IgM MACELISA. The positive predictive values for NS1 ICT and NS1 ELISA were $79.31 \%$ and $73.52 \%$ while the negative predictive values for the same components were $75.86 \%$ and $76.82 \%$ respectively. No correlation was seen between positivity of the sample for dengue infection and the duration of illness $(\mathrm{P}=0.750)$ (Table 2).

Following malaria, DF has become the second most prevalent mosquito-borne infection in recent years. DF cases have touched 40 million, as DHF cases are nearing a disconcerting several hundred thousand per year. The most endemic regions include Southeast Asia, Latin America, Asia, and the Caribbean (Raheel et al., 2011).

Presently, there is no single diagnostic assay that can diagnose all acute cases of dengue individually which is adequately sensitive and specific (Hang et al., 2009). The gold standard of laboratory diagnosis for acute dengue virus infection is virus isolation and characterization. Not only is it expensive, it takes 6-10 days in the least for the virus to replicate in cell culture or laboratory mosquitoes (Padhi et al., 2014) thereby making it least useful. 
Table.1 Comparison of result by different diagnostic assays

\begin{tabular}{|c|c|c|}
\hline Diagnostic test $(\mathbf{n = 5 3})$ & No. of dengue positive samples & Percentage \\
\hline NS1 ICT rapid card test & 29 & 54.7 \\
\hline NS1 ELISA & 34 & 64.2 \\
\hline IgM MAC-ELISA & 44 & 81.1 \\
\hline
\end{tabular}

Table.2 Comparison of duration with different dengue parameters

\begin{tabular}{|c|c|c|c|}
\hline Duration of illness & NS1 rapid positive & NS1 ELISA positive & IgM ELISA positive \\
\hline $\mathbf{1 - 5}$ days $(\mathbf{n}=\mathbf{5 2})$ & $19(36.5 \%)$ & $21(40.4 \%)$ & $25(48.1 \%)$ \\
\hline 6-10 days $(\mathbf{n}=\mathbf{6 4})$ & $10(\mathbf{1 5 . 6 \% )}$ & $13(20.3 \%)$ & $19(29.7 \%)$ \\
\hline Total $(\mathbf{n}=\mathbf{1 1 6})$ & $29(25 \%)$ & $34(29.3 \%)$ & $44(37.9 \%)$ \\
\hline
\end{tabular}

Diagnosis during the febrile phase can be achieved by the dengue virus specific RT$\mathrm{PCR}$. Although dengue IgM serology is a simple approach to diagnosis, this strictly requires paired specimens for definitive laboratory determination similar to $\operatorname{IgG}$ serology and both are not sensitive during the acute-phase of the illness. IgG in addition, lacks specificity because of cross-reactivity with other flaviviruses (Hang et al., 2009; de Oliveira Poersch et al., 2005).

A new approach to diagnosis of acute dengue is detection of secreted NS1 protein. (Dussart et al., 2008) NS1 protein is highly conservative for all the serotypes of dengue and they circulate in high levels in the blood during the first few days of the illness (Padhi et al., 2014; Tank et al., 2012) owing to their long half-life in blood (Shrivastava et al., 2011). Even with this advantage, prevalent reports regarding detection of NS1 in the presence of antibodies are conflicting in nature (Zhang et al., 2014).

Indian studies showcase prevalence rates of dengue to range from $8.39 \%-53.3 \%$ (Kumar et al., 2010; Kulkarni et al., 2011; Datta et al., 2010; Arya et al., 2011; Garg et al., 2011) and based on test results by NS1 ICT, NS1 ELISA and IgM MAC-ELISA, prevalence of dengue infection in our study was found to be $45.7 \%$. From 116 samples, detection of NS1 by ICT was $25 \%$ with $52.27 \%$ sensitivity and the same by ELISA was at $29.3 \%$ with $56.81 \%$ sensitivity making NS1 detection by ELISA a slightly more sensitive test than NS1 testing by ICT.

The detection of $28.3 \%$ dengue positives was by NS1 testing alone. A simple, highly sensitive and specific rapid dengue test not requiring instrumentation is highly desirable for wide application to confirm acute dengue especially in an outpatient setting or for application in the field. The findings of Zainah et al., (2009) show that the rapid dengue NS1 antigen immunochromatography test device meets this intended purpose. With its high specificity (99.5\%) and positive predictive value (PPV) (99.6\%), they recommend the use of rapid immunochromatography test device for diagnosing a population group defined by clinical symptomatology of acute dengue infection. In concordance, our study also saw a higher specificity of $91.66 \%$ for NS1 ICT in comparison to $87.5 \%$ for NS1 ELISA as were studies by other authors (Kulkarni et al., 2011; Chakraverti et al., 2012). PPVs for both NS1 tests were below $80 \%$ but by ICT the values were higher at $79.31 \%$ whereas ELISA 
results was marginally lower at $73.52 \%$ in agreement with Zainah et al., (2009).

Hang et al., 2009 in their study have decreed that dengue can be diagnosed in hospitalised patients in the first 3 days of illness using a single specimen by NS1 detection via ELISA or lateral flow rapid tests providing a reasonably sensitive and specific approach. In defiance of the results of the present study, no statistically significant superiority was noted in NS1 antigen detection by either the immunochromatography card or the immunosorbent assay over each other in agreement with Hang et al., (2009). Despite investigators Kulkarni et al., (2011) and Chakraverti et al., (2012) having noted that there is no necessity to repeat the test once results have been declared positive by NS1 since it is a highly specific marker of dengue infection, other authors such as Osorio et al., (2010) point out variability in their sensitivity (ranging between $37 \%$ and $98.9 \%$ ) attributable in part by the fact that sensitivity decreases with time after fever onset and in secondary infections. Addition of an IgM test alongside NS1 test improves the sensitivity by being complimentary to NS1 in such situations. (Osorio et al., 2010; Guzman et al., 2010) Overlapping dual positivity of antigen and antibody during 4-8 days of fever have been recorded. (Blacksell et al., 2008) Therefore, it is advisable to test an acute sample for both NS1 antigen and IgM antibody, more so if an acute sample is NS1 negative in suspected clinical cases of acute dengue fever.

Our findings suggest that either ICT or ELISA tests for NS1 antigen detection can be used for early diagnosis of dengue virus infection. None is superior to the other. The detection of dengue infections increased on addition of $\operatorname{IgM}$ antibody testing. We conclude that simultaneous detection of NS1 antigen and $\operatorname{IgM}$ antibodies would be potentially useful to diagnose dengue. Single tests are unreliable markers for diagnosis of dengue in acute cases. Hence, we highly recommend a battery of tests for accurate diagnosis and management of dengue cases.

\section{References}

Arya SC, Agarwal N and Parikh SC, 2011. Usefulness of detection of dengue NS1 antigen along-side $\operatorname{IgM}$ plus $\operatorname{IgG}$, and concurrent platelet enumeration during an outbreak. Trans Royal Soc Trop Med Hyg, 105: 358-359.

Blacksell SD, Mammen Jr MP, Thongpaseuth S, Gibbons RV, Jarman RG, Jenjaroen $\mathrm{K}$, et al., 2008. Evaluation of the Panbio dengue virus nonstructural 1 antigen detection and immunoglobulin $M$ antibody enzyme-linked immunosorbent assays for the diagnosis of acute dengue infections in Laos. Diagn Microbiol Infect Dis, 60:43-49.

Chakraverti TK, Malkappa SK and Saileea K, 2012. Comparison of diagnostic efficacy of NS1 antigen based immunochromatographic test with immuno-sorbent assay and its role in detection of early dengue infection. $J$ Evol Med Dental Sci, 1(6):1071-1075.

Datta S and Wattal C, 2010. Dengue NS1 antigen detection: a useful tool in early diagnosis of dengue virus infection. Indian J Med Microbiol, 28(2):107-10.

de Oliveira Poersch C, Pavoni DP, Queiroz $\mathrm{MH}$, de Borba L, Golgenberg S, dos Santos CND, et al., 2005. Dengue virus infections: comparison of methods for diagnosing the acute disease. J Clin Virol, 32:272-277.

Dussart P, Petit L, Labeau B, Bremand L, Leduc A Moua D, et al., 2008. Evaluation of two new commercial tests for the diagnosis of acute dengue virus infection using NS1 antigen detection in human serum. PLos Neg Trop Dis, 
2(8):e280.

Garg A, Garg J, Rao YK, Upadhyay GC and Sakhuja S, 2011. Prevalence of dengue among clinically suspected febrile episodes at a teaching hospital in North India. J Infect Dis Immunity, 3(5):85-89.

Guzman MG, Jaenisch T, Gaczkowski R, Hang VTT, Sekaran SD, Kroeger A, et al., 2010. Multi-country evaluation of the sensitivity and specificity of two commercially-available NS1 ELISA assays for dengue diagnosis. PLoS $\mathrm{Neg}$ Trop Dis, 4(8):e811.

Hang VT, Nguyet NM, Trung AT, Tricou V, Yoksan S, Dung NM, et al., 2009. Diagnostic Accuracy of NS1 ELISA and lateral flow rapid tests for dengue sensitivity, specificity and relationship to viraemia and antibody responses. PLoS Neg Trop Dis, 3(1):e360.

Kulkarni RD, Patil SS, Ajantha GS, Upadhya AK, Kalabhavi AS, Shubhada RM, et al., 2011. Association of platelet count and serological markers of dengue infection - importance of NS1 antigen. Indian J Med Microbiol, 29(4):359-62.

Kumar A, Rao CR, Pandit V, Shetty S, Bammigatti C, Samarasinghe CM, 2010. Clinical Manifestations and Trend of Dengue Cases Admitted in a Tertiary Care Hospital, Udupi District, Karnataka. Indian J Community Med, 35(3):386-390.

Osorio L, Ramirez M, Bonelo A, Villar LA and Parra B, 2010. Comparison of the diagnostic accuracy of commercial NS1-based diagnostic tests for early dengue infection. Virol J, 7:361.

Padhi S, Dash M, Panda P, Parida B,
Mohanty I, Sahu S, et al., 2014. Indian J Med Res, 140: 660-664.

Raheel U, Faheem M, Riaz MN, Kanwal N, Javed F, Zaidi NSS et al., 2011 Dengue fever in the Indian subcontinent: an overview. J Infect Dev Ctries, 5(4):239247.

Shrivastava A, Dask PK, Tripathi NK, Sahni AK, Gopalan N and Rao PVL, 2011. Evaluation of a commercial dengue NS1 enzyme-linked immunosorbent assay for early diagnosis of dengue infection. Indian J Med Microbiol, 29(1):51-55.

Tank AG and Jain MR, 2012. Trend of Dengue in a Tertiary Care Hospital of Surat City, Western India. National J Community Med, 3(2):302-304.

Watthanaworawit $\mathrm{W}$, Turner P, Turner CL, Tanganuchitcharnchai A, Jarman RG, Blacksell SD et al., 2011. A prospective evaluation of diagnostic methodologies for the acute diagnosis of dengue virus infection on the Thailand-Myanmar border. Trans Royal Soc Trop Med Hyg, 105:32-37.

Zainah S, Wahab AHA, Mariam M, Fauziah MK, Khairul AH, Roslina I, et al., 2009. Performance of a commercial rapid dengue NS1 antigen immunochromatography test with reference to dengue NS1 antigencapture ELISA. J Virol Methods, 155:157-160.

Zhang H, Li W, Wang J, Peng H, Che X, Chen X, et al., 2014. NS1-based tests with diagnostic utility for confirming dengue infection: a meta-analysis. Int $J$ Infect Dis, 26:57-66.

\section{How to cite this article:}

Mahesh Kumar, S. and Sheethal, S. 2018. Comparison of NS-1 Antigen Detection by ICT and ELISA for Evaluating Acute Dengue. Int.J.Curr.Microbiol.App.Sci. 7(02): 3652-3656. doi: https://doi.org/10.20546/ijcmas.2018.702.434 